\title{
Fisher Information Analysis of earthquake-related geoelectrical signals
}

\author{
L. Telesca, V. Lapenna, and M. Lovallo \\ Istituto di Metodologie per l'Analisi Ambientale, Consiglio Nazionale delle Ricerche, C.da S.Loja, 85050 Tito (PZ), Italy
}

Received: 23 May 2005 - Revised: 29 June 2005 - Accepted: 29 June 2005 - Published: 29 July 2005

Part of Special Issue "Seismic hazard evaluation, precursory phenomena and seismo electromagnetics"

\begin{abstract}
We studied the time fluctuations in the dynamics of geoelectrical data, recorded in Tito site, which is located in a seismic area of southern Italy. We used the Fisher Information Measure, which is a powerful tool to investigate complex and nonstationary signals. The time evolution of the Fisher Information Measure calculated for our signal reveals links with the earthquakes occurring in the investigated area.
\end{abstract}

\section{Introduction}

We present an approach to analyze earthquake-related geoelectrical signals, by means of the Fisher Information Measure (FIM), which was introduced by Fisher in 1925 in the context of statistical estimation (Fisher, 1925). In a seminal paper Frieden has shown FIM to be a versatile tool to describe the evolution laws of physical systems (Frieden, 1996). FIM permits to accurately describe the behavior of dynamic systems, and to characterize the complex signals generated by these systems (Vignat and Bercher, 2003). This approach has been used by Martin et al. to characterize the dynamics of EEG signals (Martin et al., 1999). Martin et al. have shown the informative content of FIM in detecting significant changes in the behavior of nonlinear dynamical systems (Martin et al., 2001), characterizing, thus, FIM as an important quantity involved in many aspects of the theoretical and observational description of natural phenomena.

The variability of several geophysical parameters can be used to give a more deep comprehension of the dynamics underlying tectonic processes (Park, 1997). In particular, geoelectrical signals are the result of the interaction among very heterogeneous mechanisms which can be influenced by the particular structure of the focal area (Patella et al., 1997). Several physical mechanisms for their generation have been proposed, e.g. it has been suggested that these signals are

Correspondence to: L. Telesca

(ltelesca@imaa.cnr.it) emitted when the stress reaches a critical value in the focal area (Freund, 2000). Therefore, these signals can convey useful information about the nonlinear tectonic dynamics. Earthquakes, indeed, are the phenomenology of the interaction of lithospheric processes of different physical origin and of different spatial and temporal scales. The lithosphere can be considered as a hierarchy of volumes, from tectonic plates to grains of rock. Their relative motion against the forces of friction and cohesion is realized to a large extent through earthquakes. The motion is controlled by a wide variety of independent processes, concentrated in the thin boundary zones between the volumes. A boundary zone has a similar hierarchical structure, consisting of volumes, separated by boundary zones, etc. Altogether, these processes transform the lithosphere into a large nonlinear system, featuring instability and deterministic chaos (Keilis-Borok, 1990).

In this study we analyze the FIM of a geoelectrical time series, recorded between 1 July 2004 and 9 November 2004 by station Tito, located in one of the most seismically active areas of southern Italy. The geoelectrical acquisition system consists of a vertical dipole with two no-polarizable electrodes, put into the ground at $5 \mathrm{~m}$ and $20 \mathrm{~m}$ depth respectively, to avoid external meteoclimatic effects. The mutual distance among the electrodes is, therefore, $15 \mathrm{~m}$. Figure 1 shows the signal variation. The sampling time is $1 \mathrm{~min}$. In August a gap occurred for technical problems.

\section{Fisher Information Measure}

Let us introduce the relevant Fisher-associated quantities. Let $\mathrm{f} \equiv \mathrm{q}^{2}$ be a probability density in $\mathfrak{R}^{N}(\mathrm{~N} \geq 1)$. Fisher's quantity of information associated to $f$ (or to the probability amplitude q) is defined as the (possibly infinite) non-negative number I

$I(f)=\int_{\Re^{N}} d \mathbf{x} \frac{|\nabla f|^{2}}{f}$ 


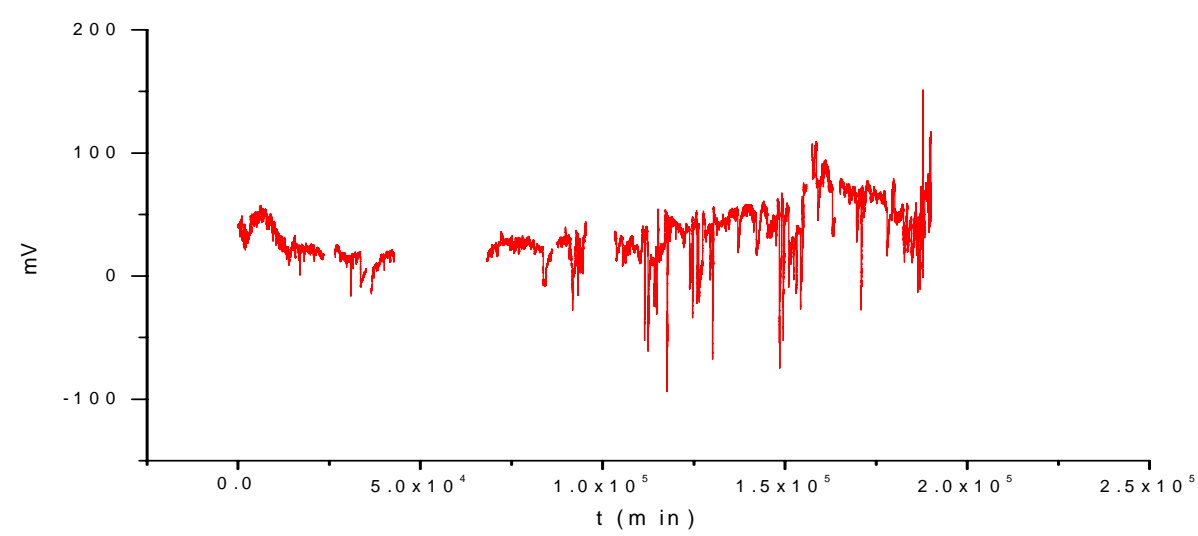

Fig. 1. Time variability of geoelectrical signal measured at station Tito, southern Italy, from 1 July 2004 to 9 November 2004.

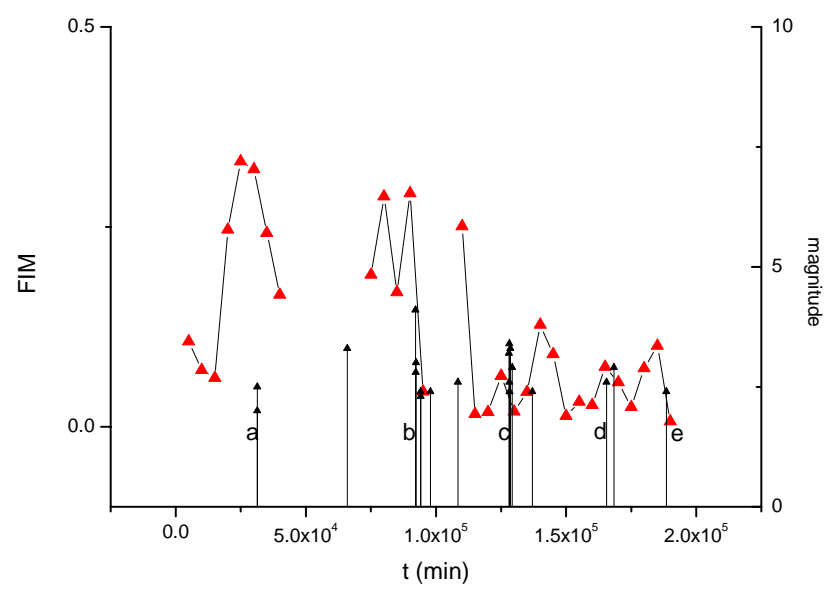

Fig. 2. Temporal variation of the FIM of Tito data; the vertical arrows indicate the occurrences of the earthquakes selected by the Dobrovolskiy's rule.

or in terms of the amplitudes

$I(q)=\int_{\Re^{N}} d \mathbf{x}(\nabla q \times \nabla q)$.

This formula defines a convex, isotropic functional I, which was first used by Fisher (1925) for statistical purposes, and plays a fundamental role in information theory. It is clear that from Eq. (2) the integrand, being the scalar product of two vectors, is independent of the reference frame (Martin et al., 2001).

Let us focus the attention on one-dimensional case. Let us consider a random variable $\mathrm{X}$ whose probability density function is denoted as $f_{X}(x)$. Its FIM is defined as

$I_{X}=\int\left(\frac{\partial}{\partial x} f_{X}(x)\right)^{2} \frac{d x}{f_{X}(x)}$.

Equation (3) involves the calculation of the probability density function (pdf) $f_{X}(x)$. A rough approximation of the unknown probability density $\mathrm{f}$ is given by the histogram. Let us consider the statistical sample by $\left\{s_{i}\right\}_{i=1}^{N}$, where $\mathrm{N}$ is the length of the sample. We consider a finite interval $[a, b]$ such that $a \leq \min _{i}\left\{s_{i}\right\}$ and $b \geq \max _{i}\left\{s_{i}\right\}$.

Next we divide the interval $[a, b]$ into $n$ nonintersecting subintervals of equal length $\mathrm{h}=(\mathrm{b}-\mathrm{a}) / \mathrm{n}$. A histogram is a function $\mathrm{f}_{N, n}(\mathrm{x})$, constant on each of the subintervals $\left[\mathrm{x}_{k}\right.$, $\left.\mathrm{x}_{k+1}\right), \mathrm{k}=1,2, \ldots, \mathrm{n}$, defined as follows:

$f_{N, n}(x)=\frac{\#\left\{s_{i} \in\left[x_{k}, x_{k+1}\right): x \in\left[x_{k}, x_{k+1}\right)\right\}}{n h}$

where $\#\{\ldots\}$ counts the number of data values falling into the specified intervals of the signal. The best convergence to the searched density function is obtained if the number of subintervals $\mathrm{n}$ is proportional to the cube root of the number $\mathrm{N}$ of observations (Mercik et al., 1999).

\section{Results}

We analyzed the time-dependent FIM or local FIM. By using the concept of sliding window (Gamero et al., 1997; Martin et al., 2000) one can calculate the temporal evolution of the local FIM. In our case we divided the whole series into nooverlapping windows. The duration of each window was set to $5 \times 10^{3} \mathrm{~min}$. The choice of this particular duration is almost subjective, but it represents a good agreement between the amount of data in each window to estimate the FIM and the total number of windows to evaluate a time variability of the FIM. We calculated the local FIM in each window by means of the procedure given by Eqs. (3) and (4), only if the number of points in the window is larger than $60 \%$ of the length of the window (that is, 3000 points), in order to have sufficient amount of points to estimate the histogram. Figure 2 shows the variation of the local FIM. It is evidently a variability in the time pattern of the FIM, which denotes that the geoelectrical system changes between disordered states (low FIM values) and ordered ones (high FIM values). The most striking feature of the FIM time pattern is the quasi-spike-like behavior, which indicates that the geoelectrical system almost suddenly changes its status. 
Since geoelectrical signals could be aslo connected with seismogenetic mechanisms (Patella et al., 1997; Freund, 2000), we investigated a possible correlation between the FIM time pattern and earthquakes. In particular, the quasispike-like behavior of the FIM time pattern can be put in relation with seismic phenomena. Therefore, in the same plot there are also shown the earthquakes occurring in the area investigated and during the observation period. The earthquakes have been selected satisfying the Dobrovolskiy's rule (Dobrovolskiy, 1993; Dobrovolskiy et al., 1979). This law, which is a theoretical relation between earthquake magnitude, distance from the epicenter and volumetric strain, states that detectable seismically induced strain exceeds $10^{-8}$. From this relation the maximum distance from the epicenter in which the effects of the earthquake are detectable is $\mathrm{r}=10^{0.43 \mathrm{M}}$, where $\mathrm{r}$ is measured in $\mathrm{km}$ and $\mathrm{M}$ is the magnitude of the earthquake. We selected 21 earthquakes occurring during the observation period. The quasi-spike-like behavior of the FIM is given by the presence of local maxima or "anomalous" FIM values. From Fig. 2, we observe that there is an enhancement of the FIM values in correspondence to the earthquakes. In particular, the earthquakes labeled by the letters a-e occur after the increase of the FIM.

\section{Discussion and conclusions}

The geophysical phenomenon underlying the geoelectrical variations is complex and the physical laws that govern the process are not completely known. The most known theory is based on the electrokinetic hypothesis. The electrokinetic currents can be observed in water-saturated media with fluidfilled channels (Mizutani et al., 1976). The walls of pores and cracks in a solid body generally adsorb cations from the liquid. Moving along the channel, the liquid carries ions of opposite sign, and thus produces an extrinsic electric current (Mizutani et al., 1976). In a seismic focal region the increasing accumulation of strain can cause dilatancy of rocks (Nur, 1972). The phenomenon of dilatancy consists in the formation and propagation of cracks inside a rock as stress reaches a critical value (Brace et al., 1966). If the rocks in the focal region and surrounding volumes are saturated with fluids, the voids generate pressure gradients. Hence, fluids invade the newly opened voids and flow until the pressure balances inside the whole system of interconnected pores. During the fluid invasion the condition of rock weakening can be reached and the earthquake is triggered. Furthermore, it has been recently given a theoretical analysis of the influence of possible fractal structure in earthquake hypocenter zone on earthquake-related geoelectrical phenomena, where fracture zone with space-variable porosity is considered as a model of an earthquake hypocenter zone in which the electrokinetic current results from fluid filtration in a fractal pore network (Surkov et al., 2002).

Our analysis has suggested that geoelectrical signals measured in seismic areas are characterized by significant changes in the variability of the FIM. These changes can be induced by seismogenic processes (leading to detectable earthquakes or crustal deformations). In particular, during seismic activity the FIM tends to increase, indicating a tendency of the whole system to behave more orderly. Following Martin et al. (2001), high FIM values indicate the incoming of regular dynamics, while low FIM values denote the presence of chaos-like behavior in the system. During periods of seismic activity, fresh cracks appear close; because of the pressure release due to cracking, they are under lower pressure, so that water from uncracked outer region can penetrate into them as soon as a network of connected channels or fractal clusters is formed. The closed fresh cracks may be considered as the sink of water from surrounding higher pressure areas (Surkov et al., 2002). Such crack organization can justify a larger regularity in the geoelectrical variability, during the acting of seismogenic processes.

We suggested a new approach in investigating the correlation between the temporal fluctuations of geoelectrical signals and earthquakes, on the basis of the Fisher Information Measure, which acts as a detector of changes in the dynamical behavior of the system generating those data. The particular observed pattern, modulated by the occurrence of the earthquakes, suggests that the signal varies between sets of disordered states (small FIM) and sets of ordered states (large FIM) (Frieden, 1996).

The use of FIM in investigating the temporal fluctuations of geoelectrical signals can lead to a better understanding of such complexity. The variation of the FIM of the time series has been investigated suggesting some correlation between the increases of the local FIM with earthquakes.

Edited by: P. F. Biagi

Reviewed by: two referees

\section{References}

Brace, W. F., Paulding, B. W., and Sholz, C. H.: Dilatancy in the fracture of cristalline rocks, J. Geophys. Res., 71, 3939-3953, 1966.

Dobrovol'skiy, I. P.: Analysis of preparation of a strong tectonic earthquake, Phys. Solid Earth, 28, 481-492, 1993.

Dobrovol'skiy, I. P., Zubkov, S. I., and Miachkin, V. I.: Estimation of the size of earthquake preparation zones, Pageoph 117, 10251044, 1979.

Fisher, R. A.: Theory of statistical estimation, Proc. Cambridge Philos. Soc., 22, 700R-725R, 1925.

Freund, F.: Time-resolved study of charge generation and propagation in igneous rocks, J. Geophys. Res. 105(B5), 11001-11020, 2000.

Frieden, B. R.: Fisher information as a measure of time, Astrophys. Space Sci., 244, 387-391, 1996.

Gamero, L., Plastino, A., and Torres, M. E.: Wavelet analysis and nonlinear dynamics in a nonextensive setting, Physica A, 246, 487-509, 1997.

Keilis-Borok, V. I.: The lithosphere of the earth as nonlinear system with implications for earthquake prediction, Rev. Geophys., 28, 19-34, 1990. 
Martin, M. T., Pennini, F., and Plastino, A.: Fisher's information and the analysis of complex signals, Phys. Lett. A, 256, 173-180, 1999.

Martin, M. T. Perez, J., and Plastino, A.: Fisher information and nonlinear dynamics, Physica A, 291, 523-532, 2001.

Martin, M. T. Plastino, A. R., and Plastino, A.: Tsallis-like information measures and the analysis of complex signals, Physica A, 275, 262-271, 2000.

Mercik, S., Weron, K., and Siwy, Z.: Statistical analysis of ionic current fluctuations in membrane channels, Phys. Rev. E, 60, 7343-7348, 1999.

Mizutani, H., Ishido, T., Yokokura, T., and Ohnishi, S.: Electrokinetic phenomena associated with earthquakes, Geophys. Res. Lett., 3, 365-368, 1976.
Nur, A.: Dilatancy pore fluids and premonitory variations of ts/tp travel times, Bull. Seism. Soc. Am., 62, 1217-1222, 1972.

Park, S. K.: Monitoring resistivity change in Parkfield, California: 1988-1995, J. Geophys. Res., 102, 24 545-24 559, 1997.

Patella D., Tramacere A., and Di Maio, R.: Modelling earth current precursors in earthquake prediction, Annali di Geofisica, 40, 495-517, 1997.

Surkov, V. V. Uyeda, S. Tanaka, H., and Hayakawa, M.: Fractal properties of medium and seismoelectric phenomena, J. Geodynamics, 33, 477-487, 2002.

Vignat, C., and Bercher, J.-F.: Analysis of signals in the FisherShannon information plane, Phys. Lett. A, 312, 27-33, 2003. 